\title{
Joint action with European CRSwNP Patients for better
}

\section{Outcomes}

The October 2019 issue of Rhinology provides the Rhinology community with an overview of the latest information on disease burden, patients' perspectives and partnership opportunities, novel diagnostics and novel approaches for better outcomes in rhinosinusitis care. The Rhinology specialty is clearly on the move, with research groups worldwide paving the way for a better future of CRS care.

Given the underestimation of the disease impact and the significant number of patients being uncontrolled despite guideline-based treatment, the need of highlighting the burden of CRSwNP is mandatory ${ }^{(1)}$. Within the October 2019 issue of Rhinology, the Ga2len research consortium confirms the major impact of CRSwNP on quality of life, underscoring the need for better outcomes of currently available treatment strategies and/or need for novel treatments with better outcomes. A European CRS and Asthma patient advisory board is launched in 2019, with clear objectives for the future (Pugin et al.) building further on initiatives to increase patient empowerment ${ }^{(2)}$. Indeed, CRS patients can help the Rhinology community moving forward in a better way by joint actions in advocacy, education, therapy adherence and even prevention (3). The research team of Carl Philpott has explored patients' perspectives on objective trial parameters leading to better cooperation with and retention in trials, which builds further on the recently published CHROME document ${ }^{(4)}$. In the context of better understanding the immune profile of CRSWNP and endotype-driven treatment ${ }^{(5)}$, Biggs et al. report on the immunological profile of CRSwNP patients with Th2/Th17 cytokines release by CRSwNP tissue from both polyp and non-polyp sinus tissue of the same patient. Following up on previous work on gustometric taste sensitivity by the group of Hummel et al. ${ }^{(6,7)}$, forced and non-forced gustatory testing outcomes are diffe- rent for bitter taste (Besser et al.). Huart et al. have developed a novel trigeminal chemosensory function test, based on the Sniffin' sticks principle and building further on their experience in the field of finetuning the olphactory and chemosensory function of the nose ${ }^{(9)}$. These results pave the way for better understanding and treatment of trigeminal chemonsensory nerve dysfunction in relation to ageing and disease. In addition, effect of gender on odor identification seem present only in individuals under 50 years of age according to the report by Zou et al. The research team of Riechelmann here reports on a the promising outcomes of novel phytotherapeutic formulation in acute rhinosinusitis, paving novel opportunities for treatment of bothersome acute rhinosinusitis.

The novel trends in CRS care are also reflected in the approach of allergic rhinitis, with similar principles of Precision Medicine (9) being advocated for daily practice in allergic rhinitis care during the annual Master Class on Allergic Rhinitis organized by ARIA / EUFOREA in Brussels.

The Rhinology Editorial team hopes you will get inspired by reading the October 2019 issue of Rhinology as much as they did, and get motivated to proceed and do better for patients suffering from CRSwNP!

by Peter Hellings

Leuven, Belgium

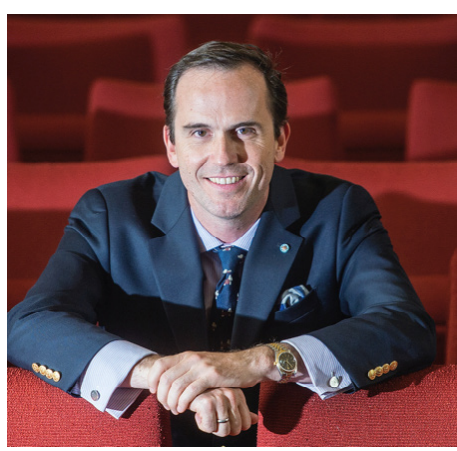

\section{References}

1. Fokkens WJ, Bachert C, Bernal-Sprekelsen $M$, et al. Rhinology Future Debates, an EUFOREA Report. Rhinology. 2017;55(4):298-304.

2. Seys SF, Bousquet J, Bachert C, et al. mySinusitisCoach: patient empowerment in chronic rhinosinusitis using mobile technology. Rhinology. 2018;56(3):209-215.

3. Hopkins C, Surda P, Bast F, Hettige R, Walker A, Hellings PW. Prevention of chronic rhinosinusitis. Rhinology. 2018;56(4):307-315.

4. Hopkins $C$, Hettige R, Soni-Jaiswal A, et al. CHronic Rhinosinusitis Outcome MEasures
(CHROME), developing a core outcome set for trials of interventions in chronic rhinosinusitis. Rhinology. 2018;56(1):22-32.

5. De Greve G, Hellings PW, Fokkens WJ, Pugin B, Steelant B, Seys SF. Endotype-driven treatment in chronic upper airway diseases. Clin Transl Allergy. 2017;7:22.

6. Han P, Georgi M, Cuevas M, Haehner A, Gudziol V, Hummel T. Decreased electrogustometric taste sensitivity in patients with acquired olfactory dysfunction. Rhinology. 2018;56(2):158-165.

7. Walliczek-Dworschak U, Knauer CM, Murbe D, Mainka A, Hummel T. Analysis of taste function in patients before and after cochlear implant surgery. Rhinology. 2018;56(2):149-154.

8. Foguem C, Lemdani M, Huart C. Parkinson disease in eldery patients: lessons from odour detection thresholds on olfacto-trigeminal interaction. Rhinology. 2018;56(2):127-132.

9. Hellings PW, Akdis CA, Bachert C, et al. EUFOREA Rhinology Research Forum 2016: report of the brainstorming sessions on needs and priorities in rhinitis and rhinosinusitis. Rhinology. 2017;55(3):202-210. 